\title{
Bilinear recurrences and addition formulae for hyperelliptic sigma functions
}

\author{
Harry W. Braden* Victor Z. Enolskii† and Andrew N.W. Hone ${ }^{\ddagger}$
}

February 1, 2008

\begin{abstract}
The Somos 4 sequences are a family of sequences satisfying a fourth order bilinear recurrence relation. In recent work, one of us has proved that the general term in such sequences can be expressed in terms of the Weierstrass sigma function for an associated elliptic curve. Here we derive the analogous family of sequences associated with an hyperelliptic curve of genus two defined by the affine model $y^{2}=4 x^{5}+c_{4} x^{4}+$ $\ldots+c_{1} x+c_{0}$. We show that the sequences associated with such curves satisfy bilinear recurrences of order 8 . The proof requires an addition formula which involves the genus two Kleinian sigma function with its argument shifted by the Abelian image of the reduced divisor of a single point on the curve. The genus two recurrences are related to a Bäcklund transformation (BT) for an integrable Hamiltonian system, namely the discrete case (ii) Hénon-Heiles system.
\end{abstract}

\section{Introduction}

In recent work 24, one of us has considered fourth order quadratic recurrences of the form

$$
\tau_{n+2} \tau_{n-2}=\alpha \tau_{n+1} \tau_{n-1}+\beta\left(\tau_{n}\right)^{2},
$$

where $\alpha$ and $\beta$ are constant parameters. Such recurrences arise in the theory of elliptic divisibility sequences [45, 46, 41] and their generalizations, the Somos 4 sequences [39, 43. In that context, both the parameters $\alpha, \beta$ and the iterates $\tau_{n}$ are integers, or more generally take values in $\mathbb{Q}$ or a Galois extension, and in that case the sequences have applications in number theory, as they provide a potential source of large prime numbers [13, 15]. Moreover the Somos 4 sequences, defined by a recurrence of the form (1.1), provide a simple example of the Laurent phenomenon: taking the initial data $\tau_{0}, \tau_{1}, \tau_{2}, \tau_{3}$ and the parameters $\alpha, \beta$ as variables, all subsequent terms $\tau_{n}$ for $n \geq 4$ in the sequence are Laurent polynomials in these variables. Fomin and Zelevinsky have proved that this remarkable "Laurentness" property is shared by a variety of other recurrences in one and more dimensions, with applications in combinatorics and commutative algebra (see [18] and references).

In 24 the following theorem was proved:

*School of Mathematics, University of Edinburgh, James Clerk Maxwell Building, Kings Buildings, Mayfield Road, Edinburgh EH9 3JZ, U.K. E-mail: hwb@maths.ed.ac.uk

${ }^{\dagger}$ Department of Mathematics, Heriot-Watt University, Edinburgh EH14 4AS, U.K. E-mail: vze@ma.hw.ac.uk

†Institute of Mathematics and Statistics, University of Kent, Canterbury CT2 7NF, U.K. E-mail: A.N.W.Hone@kent.ac.uk 
Theorem 1. The general solution of the quadratic recurrence relation (1.1) takes the form

$$
\tau_{n}=A B^{n} \frac{\sigma\left(z_{0}+n \kappa\right)}{\sigma(\kappa)^{n^{2}}},
$$

where $\kappa$ and $z_{0}$ are non-zero complex numbers, the constants $A$ and $B$ are given by

$$
A=\frac{\tau_{0}}{\sigma\left(z_{0}\right)}, \quad B=\frac{\sigma(\kappa) \sigma\left(z_{0}\right) \tau_{1}}{\sigma\left(z_{0}+\kappa\right) \tau_{0}},
$$

and $\sigma$ denotes the Weierstrass sigma function of an associated elliptic curve

$$
y^{2}=4 x^{3}-g_{2} x-g_{3} .
$$

The values $\kappa, z_{0}$ and the invariants $g_{2}, g_{3}$ are precisely determined from the initial data $\tau_{0}, \tau_{1}, \tau_{2}, \tau_{3}$ and the parameters $\alpha, \beta$.

In the next section we summarize some facts about elliptic divisibility sequences, Somos 4 sequences and the details of the above theorem. In particular we explain how the result of Theorem 1 is connected to the second order solvable mapping

$$
f_{n+1}=\frac{1}{f_{n-1} f_{n}}\left(\alpha+\frac{\beta}{f_{n}}\right)
$$

which is a degenerate case of the type of mapping studied by Quispel, Roberts and Thompson 37. (See also 38] for some recent work on the global behaviour of real-valued solutions of such mappings.)

Remark. The case $\alpha=0$, which was excluded from the statement of the Theorem in 24, corresponds to $\kappa$ being a half period, so that $\wp(\kappa)$ is a branch point of $E$, but then the formula for $\tau_{n}$ is has a rather trivial alternating form: $\tau_{2 k}=\tau_{0}\left(\tau_{2} / \tau_{0}\right)^{k} \beta^{k(k-1) / 2}$, $\tau_{2 k+1}=\tau_{1}\left(\tau_{3} / \tau_{1}\right)^{k} \beta^{k(k-1) / 2}$. The map (1.5) is the autonomous version of the discrete Painlevé I equation (qdPI)

$$
f_{n+1}=\frac{1}{f_{n-1} f_{n}}\left(\alpha q^{n}+\frac{\beta}{f_{n}}\right),
$$

which has a continuum limit to the first Painlevé equation [32, 38]. The qdPI map (1.6) has tau-functions that yield a sequence of $q$-polynomials [23, and in the autonomous case $q=1$ this map reduces to (1.5). Matsutani has constructed some particular solutions of (1.5) using elliptic functions, and has also considered certain higher order recurrences associated with genus two hyperelliptic functions [29] (see section 3). The above Theorem guarantees that only elliptic functions are necessary to specify the general solution of the second order map (11.5).

The result of Theorem 1 can also be understood via the addition formula

$$
\frac{\sigma(z+\kappa) \sigma(z-\kappa)}{\sigma(z)^{2} \sigma(\kappa)^{2}}=\wp(\kappa)-\wp(z)
$$

for the forward and backward shifted Weierstrass sigma function in terms of the $\wp$ function (see e.g. [47]). In section 3 this leads us to derive a higher order generalization of the recurrence relation (1.1) by considering a suitable addition formula for the Kleinian hyperelliptic sigma function associated to a curve of genus two. The hyperelliptic Kleinian sigma functions are a natural extension of Weierstrass elliptic functions to the case of higher genus (see e.g. 2, 8] and references). The addition formula we consider is a special case of the generalized Frobenius-Stickelberger formula in [12, 33, which is the exact genus two 
analogue of (1.7). The main result of our considerations is to derive an eighth order bilinear recurrence whose terms are given by an analogue of the formula (1.2). As a corollary, we also derive the solution of a family of sixth order nonlinear difference equations in terms of Kleinian $\wp$ functions. The fourth section explains how this recurrence is related to the BT (integrable discretization) of a Hamiltonian system with two degrees of freedom, namely the integrable case (ii) Hénon-Heiles system; this BT first appeared in [21, 22, and was put in an algebro-geometric setting in [28]. The extension to higher genus is briefly discussed in our concluding section.

\section{Elliptic divisibility and Somos 4 sequences}

The sequence

$$
0,1,-1,-1,-1,2,1,-3,5,7, \ldots
$$

is an example of an elliptic divisibility sequence. It is obtained from the recurrence

$$
\tau_{n+2}=\frac{\tau_{n+1} \tau_{n-1}+\left(\tau_{n}\right)^{2}}{\tau_{n-2}},
$$

with initial data taken as

$$
\tau_{1}=1, \quad \tau_{2}=\tau_{3}=\tau_{4}=-1
$$

The sequence can be consistently extended backwards for negative $n \in \mathbb{Z}$, to give an antisymmetric sequence with $\tau_{-n}=-\tau_{n}$. Remarkably, despite the division by $\tau_{n-2}$ at each iteration of (2.2), the subsequent terms of the sequence are all integers, and they satisfy the divisibility property

$$
\tau_{n} \mid \tau_{m} \quad \text { whenever } \quad n \mid m .
$$

More generally Morgan Ward [45, 46] introduced a family of such antisymmetric sequences defined by recurrences of the form

$$
\tau_{n+2} \tau_{n-2}=\left(\tau_{2}\right)^{2} \tau_{n+1} \tau_{n-1}-\tau_{1} \tau_{3}\left(\tau_{n}\right)^{2},
$$

which are derived by considering sequences of rational points $n P$ on an elliptic curve $E$ over $\mathbb{Q}$. To obtain integer sequences of this kind it is required that

$$
\tau_{0}=0, \quad \tau_{1}=1, \quad \tau_{2}, \tau_{3}, \tau_{4} \in \mathbb{Z} \quad \text { with } \quad \tau_{2} \mid \tau_{4} .
$$

Using the addition law on $E$ and considering the multiples $n P=\left(x_{n}, y_{n}\right)$ of a single point $P$, Ward derived the bilinear recurrence (2.4) for $\tau_{n}$, with the general term being written in terms of the sigma function associated with the curve $E$, as

$$
\tau_{n}=\frac{\sigma(n \kappa)}{\sigma(\kappa)^{n^{2}}} .
$$

(See also [16, 41].)

Using the addition formula (1.7) for the Weierstrass sigma function, it is a simple exercise to use the formula (2.5) in order show that the terms of the elliptic divisibility sequence satisfy the Hankel determinant relation

$$
\tau_{n+m} \tau_{n-m}=\left|\begin{array}{ll}
\tau_{m} \tau_{n-1} & \tau_{m-1} \tau_{n} \\
\tau_{m+1} \tau_{n} & \tau_{m} \tau_{n+1}
\end{array}\right|,
$$

for all $m, n \in \mathbb{Z}$. Starting from the Hankel determinant formula, it is then easy to prove by induction that all $\tau_{n}$ are integers with the divisibility property (2.3). 
If we consider the same recurrence (2.2) but instead take initial data $\tau_{0}=\tau_{1}=\tau_{2}=\tau_{3}=$ 1 , then we find the sequence of integers

$$
1,1,1,1,2,3,7,23,59,314, \ldots
$$

known as the Somos 4 sequence (see [39, 42]). (In fact this Somos sequence is just obtained by selecting the odd index terms of the elliptic divisibility sequence (2.1), up to an alternating sign.) More generally, following the terminology of [39, 43], we refer to any sequence defined by a bilinear recurrence of the form (1.1) as a Somos 4 sequence, while the particular sequence above is denoted Somos (4). It turns out that any such sequence is associated to a sequence of points $P_{0}+n P$ on an associated elliptic curve $E$ : this fact was proved by algebraic means in the thesis of Swart [43], which refers to unpublished results established independently by both Nelson Stephens and Noam Elkies. In [24, one of us gave an alternative complex analytic proof, leading to the construction of the functional form (1.2) of the general term, as in Theorem 1 above.

The approach taken in 24] was to regard equation (1.1) as the bilinear form of an integrable map, analogous to the bilinear equation satisfied by the tau function for a soliton equation [19, 31, and then solve the initial value problem for the bilinear equation with specified initial data $\tau_{0}, \tau_{1}, \tau_{2}, \tau_{3}$ and parameters $\alpha, \beta$. The quantity $\tau_{n}$ may be regarded as being the tau function for the second order nonlinear map (1.5), to which it is related by the substitution

$$
f_{n}=\frac{\tau_{n+1} \tau_{n-1}}{\left(\tau_{n}\right)^{2}}
$$

The map (1.5) has a first integral, given by

$$
J:=J\left(f_{n-1}, f_{n}\right)=f_{n-1} f_{n}+\alpha\left(\frac{1}{f_{n-1}}+\frac{1}{f_{n}}\right)+\frac{\beta}{f_{n-1} f_{n}}=J\left(f_{n}, f_{n+1}\right) .
$$

The algebraic formula for $J$ itself implies that the pair $\left(f_{n-1}, f_{n}\right)$ lies on an elliptic curve for all $n$. In fact the general solution of the recurrence can be written in terms of the Weierstrass $\wp$ function for a curve in the canonical form (1.4), as

$$
f_{n}=\wp(\kappa)-\wp\left(z_{0}+n \kappa\right) .
$$

The construction of this curve $E$ and the points $P_{0}, P \in E$ solves the initial value problem for (1.5) which then yields the solution (1.2) for the recurrence (1.1). It is convenient for us to summarize the results of [24] by expressing the solution of this initial value problem in the form of an algorithm, as follows:

Step 1: Find the backwards iterate $\tau_{-1}$ from the initial data, evaluate the quantities $f_{0}=$ $\tau_{1} \tau_{-1} /\left(\tau_{0}\right)^{2}$ and $f_{1}=\tau_{2} \tau_{0} /\left(\tau_{1}\right)^{2}$, and use these to calculate the integral

$$
J=J\left(f_{0}, f_{1}\right)=\wp^{\prime \prime}(\kappa)
$$

Step 2: Use $J, \alpha, \beta$ to calculate

$$
\lambda=\frac{1}{3 \alpha}\left(\frac{J^{2}}{4}-\beta\right)=\wp(\kappa) .
$$

This gives the point $P=(\lambda, \mu)=\left(\wp(\kappa), \wp^{\prime}(\kappa)\right) \in E$, with $\mu= \pm \sqrt{\alpha}$.

Step 3: Construct the invariants $g_{2}, g_{3}$ of the curve $E$ as in (1.4), from the formulae

$$
g_{2}=12 \lambda^{2}-2 J, \quad g_{3}=4 \lambda^{3}-g_{2} \lambda-\alpha .
$$


Step 4: Iterate (1.5) backwards to obtain $f_{-1}$ from $f_{0}$ and $f_{1}$. Hence find the point $P_{0}=$ $(\nu, \xi)=\left(\wp\left(z_{0}\right), \wp^{\prime}\left(z_{0}\right)\right) \in E$ from the formulae

$$
\nu=\lambda-f_{0}, \quad \xi=\frac{f_{0}^{2}\left(f_{1}-f_{-1}\right)}{\mu} .
$$

Step 5: Calculate the values $\kappa, z_{0} \in \mathbb{C}$ from the elliptic integrals

$$
\kappa= \pm \int_{\infty}^{P} \frac{\mathrm{d} x}{y}, \quad z_{0}= \pm \int_{\infty}^{P_{0}} \frac{\mathrm{d} x}{y} ;
$$

these should be interpreted as the points in the Jacobian $\operatorname{Jac}(E)$ corresponding to the points $P, P_{0} \in E$ respectively. Note that because of the involution $y \rightarrow-y$ these values are only defined by to an overall \pm sign, subject to the constraint that $\wp^{\prime}(\kappa) \wp^{\prime}\left(z_{0}\right)=\xi \mu=f_{0}^{2}\left(f_{1}-f_{-1}\right)$ as in Step 4. Once $z_{0}$ and $\kappa$ are obtained then $A$ are $B$ are found from the formulae (1.3).

Remarks. It is useful to note that the coefficients $\alpha, \beta$ in the recurrence are given as elliptic functions of $\kappa$ by

$$
\alpha=\wp^{\prime}(\kappa)^{2}, \quad \beta=\wp^{\prime}(\kappa)^{2}(\wp(2 \kappa)-\wp(\kappa)) .
$$

The above solution of the initial value problem establishes an exact correspondence between two sets of six parameters: the parameters $g_{2}, g_{3}, \kappa, z_{0}, A, B$ that specify the elliptic curve $E$, the two points $P, P_{0} \in E$, and the prefactors in (1.2); and the parameters $\alpha, \beta, \tau_{0}, \tau_{1}, \tau_{2}, \tau_{3}$ specifying the constant coefficients and initial data for the recurrence (1.1). In order to interpret (1.5) as an integrable map, it is necessary to further specify a symplectic structure [6. 44]; symplectic coordinates and a Lax pair were given in [24], which make (1.5) equivalent to the discrete $g=1$ odd Mumford system in [28].

As an example of the above algorithm, we present the results for the Somos (4) sequence (2.7), with $\alpha=\beta=\tau_{0}=\tau_{1}=\tau_{2}=\tau_{3}=1$. We find $\tau_{-1}=2$, so $f_{0}=2, f_{1}=1$ gives $J=4 \mathrm{in}$ Step 1. In Steps 2 and 3 we have $\lambda=1$, set $\mu=1$ and then find $g_{2}=4, g_{3}=-1$, and in Step 4 we obtain $f_{-1}=3 / 4$ so that $\nu=-1, \xi=1$. Thus the Somos (4) sequence corresponds to the sequence of points $P_{0}+n P=(-1,1)+n(1,1)$ on the curve

$$
E: \quad y^{2}=4 x^{3}-4 x+1 \text {. }
$$

Finally, evaluating the elliptic integrals and sigma functions to 9 decimal places using the MAPLE computer algebra package (version 8), we find that the curve has real and imaginary half-periods $\omega_{1}=1.496729323$ and $\omega_{3}=1.225694691 i$ respectively, while

$$
\begin{gathered}
\kappa-2 \omega_{1}=-\int_{1}^{\infty}\left(4 t^{3}-4 t+1\right)^{-\frac{1}{2}} d t=-1.134273216, \\
z_{0}-2 \omega_{3}=\int_{-1}^{\infty}\left(4 t^{3}-4 t+1\right)^{-\frac{1}{2}} d t=0.204680500-1.225694691 i,
\end{gathered}
$$

which yield the other quantities in (1.2) as

$$
\begin{gathered}
\sigma(\kappa)=1.555836426, \quad A=\frac{1}{\sigma\left(z_{0}\right)}=0.112724016-0.824911687 i \\
B=\frac{\sigma(\kappa) \sigma\left(z_{0}\right)}{\sigma\left(z_{0}+\kappa\right)}=0.215971963+0.616028193 i
\end{gathered}
$$


However, the sequence of arguments of the sigma function can be written more succinctly as

$$
z_{0}+n \kappa \equiv(2 n-3) \hat{z}_{0}, \quad \hat{z}_{0}=0.929592715+\omega_{3}
$$

so that the iterates of the recurrence correspond to the sequence of points $(2 n-3) \tilde{P}$ on the curve E, where $\tilde{P}=(0,1), P=2 \tilde{P}$. The full sequence of points $n \tilde{P}$ is associated with the elliptic divisibility sequence (2.1).

Elliptic divisibility sequences are currently of considerable interest due to the fact that large prime numbers can occur therein (i.e. $\tau_{p}$ may be prime when the index $p$ is prime, see [13, 15, 41]). Cantor has considered the division polynomials for odd hyperelliptic curves [1], corresponding to sequences of divisors $n(P-\infty)$, which also satisfy higher order recurrences written in terms of Hankel determinants; Matsutani has obtained the functional form of these division polynomials in genus two 30. In the next section we shall derive an eighth order bilinear recurrence associated with the sequence of divisors $\mathcal{D}_{0}+n(P-\infty)$, where $\mathcal{D}_{0}=\left(P_{1}-\infty\right)+\left(P_{2}-\infty\right)$ is the reduced divisor of two points on a hyperelliptic curve of genus two.

\section{Addition of one point in genus two}

Let us consider an algebraic curve $X$ of genus two defined by the affine model

$$
X:=\left\{(x, y) \mid y^{2}=f(x) \equiv 4 x^{5}+\sum_{j=0}^{4} c_{j} x^{j}\right\},
$$

which realizes the curve as a two-sheeted covering of the Riemann sphere with $2 g+1$ branch points in the complex plane plus a single branch point $\infty$ at infinity. The vectors of canonical holomorphic differentials and canonical meromorphic (second kind) differentials are denoted

$$
\mathrm{d} \mathbf{u}=\left(\begin{array}{c}
\frac{\mathrm{d} x}{y} \\
\frac{x \mathrm{~d} x}{y}
\end{array}\right), \quad \mathrm{d} \mathbf{r}=\left(\begin{array}{c}
\frac{\left(12 x^{3}+2 c_{4} x^{2}+c_{3} x\right) \mathrm{d} x}{4 y} \\
\frac{x^{2} \mathrm{~d} x}{y}
\end{array}\right)
$$

respectively. If we let $\left(A_{1}, A_{2} ; B_{1}, B_{2}\right)$ denote the canonical homology basis for the compact Riemann surface corresponding to $X$, with non-vanishing intersections $A_{j} \cdot B_{k}=\delta_{j k}$, then the $2 \times 2$ matrices of $A$ - and $B$-periods are given by

$$
2 \omega=\left(\begin{array}{cc}
\oint_{A_{1}} \frac{\mathrm{d} x}{y} & \oint_{A_{2}} \frac{\mathrm{d} x}{y} \\
\oint_{A_{1}} \frac{x \mathrm{~d} x}{y} & \oint_{A_{2}} \frac{x \mathrm{~d} x}{y}
\end{array}\right), \quad 2 \omega^{\prime}=\left(\begin{array}{cc}
\oint_{B_{1}} \frac{\mathrm{d} x}{y} & \oint_{B_{2}} \frac{\mathrm{d} x}{y} \\
\oint_{B_{1}} \frac{x \mathrm{~d} x}{y} & \oint_{B_{2}} \frac{x \mathrm{~d} x}{y}
\end{array}\right) .
$$

The Jacobian of $X$ is the complex torus $\operatorname{Jac}(X)=\mathbb{C}^{2} / \Gamma$, where $\Gamma=2 \omega \mathbb{Z}^{2} \oplus 2 \omega^{\prime} \mathbb{Z}^{2}$ is the lattice generated by the periods of canonical holomorphic differentials. The elements $\left(P_{1}, P_{2}\right)$ of the symmetric product $(X)^{2}$ can be identified with degree zero divisors $\mathcal{D}=$ $\left(P_{1}-\infty\right)+\left(P_{2}-\infty\right)$, which are mapped to $\operatorname{Jac}(X)$ by the Abel map:

$$
\mathbf{u}=\int_{\infty}^{P_{1}} \mathrm{~d} \mathbf{u}+\int_{\infty}^{P_{2}} \mathrm{~d} \mathbf{u} \in \operatorname{Jac}(X)
$$

(where here we are basing the map at $\infty$ ). 
The Kleinian sigma function $\sigma(\mathbf{u})$, which is a quasiperiodic function of $\left(u_{1}, u_{2}\right)^{T}=\mathbf{u} \in \mathbb{C}^{2}$, is the genus two analogue of the Weierstrass sigma function. The Kleinian $\zeta$ and $\wp$ functions are defined by

$$
\begin{gathered}
\zeta_{j}(\mathbf{u})=\frac{\partial \log \sigma(\mathbf{u})}{\partial u_{j}}, \quad j=1,2 \\
\wp_{j k}(\mathbf{u})=-\frac{\partial^{2} \log \sigma(\mathbf{u})}{\partial u_{j} \partial u_{k}}, \quad \wp_{j k l}(\mathbf{u})=-\frac{\partial^{3} \log \sigma(\mathbf{u})}{\partial u_{j} \partial u_{k} \partial u_{l}}, \quad j, k=1,2 .
\end{gathered}
$$

We refer the reader to other works such as [2, 8, for a detailed introduction to hyperelliptic curves, Kleinian functions and their definition in terms of Riemann theta functions (see also 4, 12, 33, 34, and references).

Vectors $\mathbf{v}$ in the theta divisor $\Theta$ in $\operatorname{Jac}(X)$ can be characterized by the fact that

$$
\sigma(\mathbf{v})=0 .
$$

We wish to take a vector $\mathbf{v} \in \Theta$ in the theta divisor, given by

$$
\mathbf{v}=\int_{\infty}^{(\lambda, \mu)} \mathrm{d} \mathbf{u}
$$

so $\mathbf{v}$ corresponds to the (reduced) divisor of a single point $(\lambda, \mu) \in X$, with $\mu^{2}=f(\lambda)$.

In [8] (Theorem 4.9) it is proved that the Baker function

$$
\Phi: \operatorname{Jac}(X) \times X \rightarrow \mathbb{C}
$$

defined for $\mathbf{u} \in \operatorname{Jac}(X)$ and $(\lambda, \mu) \in X$ by

$$
\Phi(\mathbf{u} ;(\lambda, \mu))=\frac{\sigma(\mathbf{v}-\mathbf{u})}{\sigma_{2}(\mathbf{v}) \sigma(\mathbf{u})} \exp \left(f_{\infty}^{(\lambda, \mu)} \mathrm{d} \mathbf{r}^{T} \mathbf{u}\right), \quad \mathbf{v}=\int_{\infty}^{(\lambda, \mu)} \mathrm{d} \mathbf{u}
$$

satisfies the Schrödinger equation

$$
\left(\partial_{2}^{2}-2 \wp_{22}\right) \Phi=\left(\lambda+c_{4} / 4\right) \Phi
$$

Note that we have chosen a particular normalization for the Baker function compared with [8], including the denominator $\sigma_{2}(\mathbf{v}) \neq 0$, and the principal value symbol $f$ in (3.3) denotes the fact that the integral of the meromorphic differential $\mathrm{d} \mathbf{r}$ is regularized at infinity.

Let us define two different Baker-Akhiezer functions $\Phi_{ \pm}$related by the hyperelliptic involution, as

$$
\Phi_{ \pm}=\Phi(\mathbf{u} ;(\lambda, \pm \mu))
$$

Then from the proof of Theorem 4.9 in [8] (restricting to $g=2$ ) we have that

$$
\partial_{2} \log \Phi_{ \pm}=\frac{ \pm \mu+\partial_{2} \mathcal{P}(\lambda ; \mathbf{u})}{2 \mathcal{P}(\lambda ; \mathbf{u})}
$$

where

$$
\mathcal{P}(\lambda ; \mathbf{u})=\lambda^{2}-\wp_{22}(\mathbf{u}) \lambda-\wp_{12}(\mathbf{u})
$$

is the Bolza polynomial in genus two [5]. Hence it follows that $\mathcal{P}=\mathcal{P}(\lambda ; \mathbf{u})$ satisfies the Ermakov-Pinney equation with respect to derivatives $\partial_{2}$ in the variable $u_{2}$, namely

$$
\mathcal{P}\left(\partial_{2}^{2} \mathcal{P}\right)-\frac{1}{2}\left(\partial_{2} \mathcal{P}\right)^{2}+2 V \mathcal{P}^{2}+\frac{\mu^{2}}{2}, \quad V:=-2 \wp_{22}(\mathbf{u})-\lambda-c_{4} / 4
$$


It is a well known classical result of Ermakov (see [20] for references) that the general solution of the Ermakov-Pinney equation (3.7) is just given by a product of two solutions of the Schrödinger equation $\left(\partial_{2}^{2}+V\right) \psi=0$ with Wronskian $\mu$.

Taking the difference of the \pm equations (3.5) we have

$$
\frac{W\left(\Phi_{-}, \Phi_{+}\right)}{\Phi_{+} \Phi_{-}}=\frac{\mu}{\mathcal{P}}
$$

where

$$
W\left(\Phi_{-}, \Phi_{+}\right)=\left|\begin{array}{cc}
\Phi_{-} & \Phi_{+} \\
\partial_{2} \Phi_{-} & \partial_{2} \Phi_{+}
\end{array}\right|
$$

is the Wronskian. Clearly this must be independent of $u_{2}$, but we claim that in fact this Wronskian has precisely the value $\mu$, which means that the product $\Phi_{+} \Phi_{-}=\mathcal{P}$. Rewriting this in terms of the sigma function, we can state the following result.

Proposition. The Kleinian sigma function for a hyperelliptic curve (3.1) of genus two satisfies the following formula for addition of a single point on the curve:

$$
\frac{\sigma(\mathbf{u}+\mathbf{v}) \sigma(\mathbf{u}-\mathbf{v})}{\sigma(\mathbf{u})^{2} \sigma_{2}(\mathbf{v})^{2}}=\mathcal{P}(\lambda ; \mathbf{u})
$$

In the above, $\mathbf{u} \in \operatorname{Jac}(X)$ is a generic vector in the Jacobian, $\mathbf{v} \in \Theta \subset \operatorname{Jac}(X)$ is the image of the single point $(\lambda, \mu) \in X$ under the Abel map, and $\mathcal{P}$ is the Bolza polynomial defined by (3.6).

Proof: Starting from Baker's addition theorem for genus two [2],

$$
\frac{\sigma(\mathbf{u}+\mathbf{v}) \sigma(\mathbf{u}-\mathbf{v})}{\sigma(\mathbf{u})^{2} \sigma(\mathbf{v})^{2}}=\wp_{22}(\mathbf{u}) \wp_{12}(\mathbf{v})-\wp_{12}(\mathbf{u}) \wp_{22}(\mathbf{v})+\wp_{11}(\mathbf{v})-\wp_{11}(\mathbf{u})
$$

where $\mathbf{u}, \mathbf{v}$ are generic points in $\operatorname{Jac}(X)$, and multiplying both sides by $\sigma(\mathbf{v})^{2} / \sigma_{2}(\mathbf{v})^{2}$, the result follows by taking the limit $\sigma(\mathbf{v}) \rightarrow 0$ as $\mathbf{v}$ tends to the theta divisor. It is necessary to use the fact (see e.g. 33]) that the $x$ coordinate of the point $(\lambda, \mu) \in X$ is given, in terms of derivatives of the sigma function evaluated on the theta divisor $\Theta$, by the expression

$$
\lambda=-\frac{\sigma_{1}(\mathbf{v})}{\sigma_{2}(\mathbf{v})}
$$

This follows from the fact that differentiating $\sigma(\mathbf{v})=0$ with respect to $\lambda$ gives, by the chain rule,

$$
\frac{d v_{1}}{d \lambda} \partial_{1} \sigma(\mathbf{v})+\frac{d v_{2}}{d \lambda} \partial_{2} \sigma(\mathbf{v})=\frac{\sigma_{1}(\mathbf{v})}{\mu}+\frac{\lambda \sigma_{2}(\mathbf{v})}{\mu}=0
$$

for $\mathbf{v} \in \Theta$ given by (3.2).

Remark. Enolskii and Gibbons recently calculated the exact analogue of (3.8) in genus three. The addition formula (3.8) is a special case of the generalized Frobenius-Stickelberger addition formula in genus two considered in [12, 33]. Onishi has further generalized the Frobenius-Stickelberger formula to hyperelliptic sigma functions for all genera 34, and the special case of the formula corresponding to addition of one point has been applied to the problem of construction of Wannier functions for quasi-periodic finite-gap potentials in [4].

Cantor has constructed the division polynomials for hyperelliptic curves, and obtained certain recurrence relations for them in the paper [11, where in particular an eighth order bilinear recurrence is found in genus two. Up to a suitable normalization, Matsutani has 
considered the exact analytic expression for these division polynomials, which are equivalent to the sequence of functions

$$
a_{n}=\frac{\sigma(n \mathbf{v})}{\sigma_{2}(\mathbf{v})^{n^{2}}},
$$

known as as hyperelliptic psi-functions 29, 30. In the following theorem, we present a sequence of tau-functions that generalize these psi-functions and yet satisfy the same recurrence of Somos 8 type.

Theorem 2. Define the sequence $\left\{\tau_{n} \mid n \in \mathbb{Z}\right\}$ by

$$
\tau_{n}=A B^{n} \frac{\sigma(\mathbf{u}+n \mathbf{v})}{\sigma_{2}(\mathbf{v})^{n^{2}}}
$$

where $\mathbf{u} \in \operatorname{Jac}(X)$ is a generic vector in the Jacobian of the genus two curve (3.1), $\mathbf{v} \in$ $\Theta \subset \operatorname{Jac}(X)$ is the image of the single point $(\lambda, \mu) \in X$ under the Abel map, $\sigma$ denotes the Kleinian sigma function of the curve, and $A, B$ are arbitrary constants. Then the terms of the sequence satisfy a bilinear recurrence of order 8, given by

$$
\tau_{n+4} \tau_{n-4}=\sum_{j=0}^{3} \alpha_{j} \tau_{n+j} \tau_{n-j}
$$

where the coefficients $\alpha_{j}$ (independent of $n$ ) are given by

$$
\begin{gathered}
\alpha_{1}=\frac{\sigma(6 \mathbf{v}) \sigma(3 \mathbf{v})^{2}}{\sigma(4 \mathbf{v}) \sigma(2 \mathbf{v})^{2} \sigma_{2}(\mathbf{v})^{30}}, \quad \alpha_{3}=\frac{\sigma(3 \mathbf{v}) \sigma(5 \mathbf{v})}{\sigma(2 \mathbf{v}) \sigma(4 \mathbf{v}) \sigma_{2}(\mathbf{v})^{14}} \\
\alpha_{2}=\frac{\sigma(4 \mathbf{v})^{2}}{\sigma_{2}(\mathbf{v})^{24} \sigma(2 \mathbf{v})^{2}}\left(1-\frac{\sigma(3 \mathbf{v})^{3} \sigma(5 \mathbf{v})}{\sigma(4 \mathbf{v})^{3} \sigma(2 \mathbf{v})}\right), \quad \alpha_{0}=-\sigma(6 \mathbf{v}) /\left(\sigma(2 \mathbf{v}) \sigma_{2}(\mathbf{v})^{32}\right) .
\end{gathered}
$$

Proof. Substituting the expression (3.10) into (3.11) and using Baker's formula (3.9) together with the result of the Proposition yields an expression of the form

$$
C_{0}(\mathbf{v})+C_{11}(\mathbf{v}) \wp_{11}(\mathbf{u})+C_{12}(\mathbf{v}) \wp_{12}(\mathbf{u})+C_{22}(\mathbf{v}) \wp_{22}(\mathbf{u})=0 .
$$

The three functions $\wp_{j k}(\mathbf{u}), j, k=1,2$ on $\operatorname{Jac}(X)$ are not linearly dependent (although they do satisfy a nonlinear relation [8, 12, giving the Kummer surface in $\mathbb{C P}^{3}$ ). Therefore each of the coefficients $C_{0}(\mathbf{v}), C_{11}(\mathbf{v}), C_{12}(\mathbf{v}), C_{22}(\mathbf{v})$ must vanish, which leads to a linear system for the $\alpha_{j}$ as functions of $\mathbf{v}$. This determines the above formulae for the coefficients $\alpha_{j}$ uniquely in terms of $\sigma$, its derivative $\sigma_{2}$, and the $\wp_{j k}$ evaluated at various multiples of $\mathbf{v}$. The terms involving $\wp_{j k}$ can be removed by making use of the addition formulae (3.9) and (3.8) to yield the expressions (3.12) and (3.13) in terms of $\sigma$ and $\sigma_{2}$ alone. Taking the limit $\mathbf{u} \rightarrow 0$, these are equivalent to Matsutani's expressions for the coefficients in the eighth order bilinear recurrence for the psi-function (see formula (3.13) in [29]).

Corollary. The sequence of Bolza polynomials

$$
f_{n}=\mathcal{P}(\lambda ; \mathbf{u}+n \mathbf{v})=\lambda^{2}-\wp_{22}(\mathbf{u}+n \mathbf{v}) \lambda-\wp_{12}(\mathbf{u}+n \mathbf{v}),
$$

for $\mathbf{u} \in \operatorname{Jac}(X)$ and $\mathbf{v} \in \Theta$, satisfies the sixth order nonlinear difference equation

$$
f_{n}^{4} \prod_{k=1}^{3}\left(f_{n+k} f_{n-k}\right)^{4-k}=\alpha_{0}+\sum_{j=1}^{3} \alpha_{j} f_{n}^{j} \prod_{k=1}^{j-1}\left(f_{n+k} f_{n-k}\right)^{j-k},
$$

with the coefficients $\alpha_{j}$ as given in equations (3.12), and (3.13). 
Proof of Corollary. Upon setting

$$
f_{n}=\frac{\tau_{n+1} \tau_{n-1}}{\left(\tau_{n}\right)^{2}}
$$

with $\tau_{n}$ as in (3.10), and using the addition formula (3.8), the result is an immediate consequence of Theorem 2.

Remarks. Taking $\mathbf{u}$ as the Abelian image of $\left(P_{1}, P_{2}\right) \in(X)^{2}$, the sequence (3.14) corresponds to the linear flow $\mathbf{u}+n \mathbf{v}$ in the Jacobian, or equivalently the sequence of divisors $\mathcal{D}_{n} \sim \mathcal{D}_{0}+n(P-\infty) \sim \mathcal{D}_{0}-n(\hat{P}-\infty)$ with $\mathcal{D}_{0} \sim\left(P_{1}-\infty\right)+\left(P_{2}-\infty\right)$ and $\hat{P}=(\lambda,-\mu)$, the image of $P=(\lambda, \mu)$ under the hyperelliptic involution. The Bolza polynomial leads to the solution of the Jacobi inversion problem for the curve (3.1) (see Theorem 2.2 in [8], and section 4 below), so that in particular if $\mathcal{D}_{n} \sim\left(x_{1}(n), y_{1}(n)\right)+\left(x_{2}(n), y_{2}(n)\right)-2 \infty$, then we have

$$
f_{n}=\mathcal{P}(\lambda ; \mathbf{u}+n \mathbf{v})=\left(\lambda-x_{1}(n)\right)\left(\lambda-x_{2}(n)\right), \quad y_{j}(n)=-\left.\partial_{2} \mathcal{P}(\lambda ; \mathbf{u}+n \mathbf{v})\right|_{\lambda=x_{j}}, \quad j=1,2
$$

Cantor's results in [1] concern the sequence of reduced divisors $\mathcal{D}_{n} \sim n(P-\infty)$, corresponding to the multiples of a single point on an odd hyperelliptic curve of genus $g$. In particular for $g=2$ he obtains a bilinear recurrence of order 8 , which is the degenerate case $\mathbf{u}=0$ $\left(P_{1} \rightarrow \infty, P_{2} \rightarrow \infty\right)$ of our construction, while the analytic derivation in that case appears in the work of Matsutani [29, 30]. The sixth order difference equation (3.15) appears as equation (3.15) in [29], where the special solutions with $\mathbf{u}=0$ are also presented.

The sigma functions of genus $g$ odd hyperelliptic curves, given by $y^{2}=f(x)$ with $f$ a polynomial of odd degree $2 g+1$, are known to be tau functions of the Korteweg-deVries $(\mathrm{KdV})$ hierarchy of partial differential equations (see $[8]$ for instance). It is also known that when the curve degenerates completely to $y^{2}=4 x^{2 g+1}$, the corresponding sigma function degenerates to a polynomial (see [9, 34]), which gives a rational solution of KdV in terms of a Schur function (see [1] and chapter 14 in [26]). It is instructive to consider the case when the curve (3.1) for $g=2$ degenerates to a singular rational curve:

$$
y^{2}=4 x^{5} .
$$

In that case the Kleinian sigma function degenerates to the Schur function

$$
\sigma(\mathbf{u})=u_{1}-\frac{u_{2}^{3}}{3}
$$

which is the tau function of the three-pole rational solution of the KdV equation

$$
4 \partial_{1} V=\partial_{2}^{3} V+6 V \partial_{2} V, \quad V=-2 \wp_{22}(\mathbf{u})=2 \partial_{2}^{2} \log \sigma(\mathbf{u}) .
$$

The theta divisor consists of vectors of the form

$$
\mathbf{v}=\left(\begin{array}{c}
\int_{\infty}^{(\lambda, \mu)} \frac{\mathrm{d} x}{\sqrt{4 x^{5}}} \\
\int_{\infty}^{(\lambda, \mu)} \frac{x \mathrm{~d} x}{\sqrt{4 x^{5}}}
\end{array}\right)=\left(\begin{array}{c}
-\frac{1}{3} \lambda^{-3 / 2} \\
-\lambda^{-1 / 2}
\end{array}\right) \equiv\left(\begin{array}{c}
\gamma^{3} / 3 \\
\gamma
\end{array}\right), \quad \gamma \in \mathbb{C},
$$

satisfying $\sigma(\mathbf{v})=0$. It is trivial to check that the Schur function satisfies the addition formula (3.8). Defining $\tau_{n}$ in terms of the Schur function (3.16) by (3.10), it is easy to verify that this gives a particular solution of the eighth order recurrence (3.11) with

$$
\alpha_{0}=-\frac{35}{\gamma^{64}}, \quad \alpha_{1}=\frac{56}{\gamma^{60}}, \quad \alpha_{2}=-\frac{28}{\gamma^{48}}, \quad \alpha_{3}=\frac{8}{\gamma^{28}} .
$$




\section{BT for the case (ii) Hénon-Heiles system}

The integrable case (ii) Hénon-Heiles system is a system of two degrees of freedom defined by the natural Hamiltonian

$$
h_{1}=\frac{1}{2}\left(p_{1}^{2}+p_{2}^{2}\right)+q_{2}^{3}+\frac{1}{2} q_{2} q_{1}^{2}-\frac{1}{2} a q_{1}^{2}+c q_{2}-\frac{m^{2}}{2 q_{1}^{2}}
$$

The coordinates $q_{j}$ and momenta $p_{j}$ are canonically conjugate, and Hamilton's equations

$$
\frac{\mathrm{d} q_{j}}{\mathrm{~d} t}=\left\{h_{1}, q_{j}\right\}, \quad \frac{\mathrm{d} p_{j}}{\mathrm{~d} t}=\left\{h_{1}, p_{j}\right\}, \quad j=1,2
$$

are equivalent to the ordinary differential equation for travelling wave solutions of the fifth order flow in the KdV hierarchy [17]. The equations of motion (4.2) can be written in the form of a Lax equation

$$
\frac{\mathrm{d} \mathbf{L}}{\mathrm{d} t}=[\mathbf{N}, \mathbf{L}]
$$

where the Lax matrix $\mathbf{L}$ is

$$
\mathbf{L}\left(x ; q_{j}, p_{j}\right)=\left(\begin{array}{cc}
\frac{1}{2} p_{2} x-\frac{1}{8} p_{1} q_{1} & \mathcal{B}\left(x ; q_{j}, p_{j}\right) \\
2 x^{2}+\left(q_{2}+2 a\right) x-\frac{1}{8} q_{1}^{2} & -\frac{1}{2} p_{2} x+\frac{1}{8} p_{1} q_{1}
\end{array}\right)
$$

with

$$
\mathcal{B}\left(x ; q_{j}, p_{j}\right)=2 x^{3}+\left(-q_{2}+4 a\right) x^{2}+\frac{1}{8}\left(q_{1}^{2}+4 q_{2}^{2}-a q_{2}+16 a^{2}+4 c\right) x+\frac{1}{8}\left(p_{1}^{2}-\frac{m^{2}}{q_{1}^{2}}\right) .
$$

(Note that we have $\mathbf{L} \rightarrow-\frac{(u-a)}{8} \mathbf{L}, u \rightarrow x+a$ compared with reference [21].) The Lax equation is the compatibility condition for the linear system

$$
\mathbf{L} \Psi=y \Psi, \quad \frac{\mathrm{d} \Psi}{\mathrm{d} t}=\mathbf{N} \Psi, \quad \mathbf{N}=\left(\begin{array}{cc}
0 & x+a-q_{2} \\
1 & 0
\end{array}\right) .
$$

The genus two spectral curve is of the precise form (3.1), namely

$$
\operatorname{det}(\mathbf{L}-y \mathbf{1})=y^{2}-4 x^{5}-12 a x^{4}-\left(c+12 a^{2}\right) x^{3}-\frac{1}{2} h_{1} x^{2}-\frac{1}{2} h_{2} x-\frac{m^{2}}{64}=0,
$$

with

$$
h_{2}=\frac{1}{4}\left(q_{2}+2 a\right) p_{1}^{2}-\frac{1}{4} q_{1} p_{1} p_{2}-\frac{1}{32} q_{1}^{4}-\frac{1}{8}\left(q_{2}^{2}-2 a q_{2}+c+4 a^{2}\right) q_{1}^{2}-\frac{m^{2}\left(q_{2}+2 a\right)}{4 q_{1}^{2}}
$$

being the second independent integral, in involution with $h_{1}$ i.e. $\left\{h_{1}, h_{2}\right\}=0$. The integral $h_{2}$ generates a second commuting flow

$$
\frac{\mathrm{d} q_{j}}{\mathrm{~d} s}=\left\{h_{2}, q_{j}\right\}, \quad \frac{\mathrm{d} p_{j}}{\mathrm{~d} s}=\left\{h_{2}, p_{j}\right\}, \quad j=1,2 .
$$

Up to a shift of origin, the time variables $s, t$ can be identified with the coordinates $u_{1}, u_{2}$ respectively on $\operatorname{Jac}(X)$. Using the results of Theorem 2.2 in [8], the solution of the Hénon-Heiles system can be reduced to the Jacobi inversion problem

$$
\left(\begin{array}{c}
s \\
t
\end{array}\right) \equiv \mathbf{u}=\int_{\infty}^{\left(x_{1}, y_{1}\right)} \mathrm{d} \mathbf{u}+\int_{\infty}^{\left(x_{2}, y_{2}\right)} \mathrm{d} \mathbf{u}
$$


where the separation coordinates $x_{1}, x_{2}$ are found from the $(2,1)$ entry in the Lax matrix (4.3), given as a multiple of the Bolza polynomial by

$$
2 x^{2}+\left(q_{2}+2 a\right) x-\frac{1}{8} q_{1}^{2}=2\left(x-x_{1}\right)\left(x-x_{2}\right)=2 \mathcal{P}(x ; \mathbf{u}) .
$$

The separation variables $\left(x_{j}, y_{j}\right), j=1,2$ correspond to the reduced divisor $\mathcal{D}_{0}=\left(x_{1}, y_{1}\right)+$ $\left(x_{2}, y_{2}\right)-2 \infty$, and they are related to the Kleinian functions by

$$
\wp_{12}(\mathbf{u})=-x_{1} x_{2}, \quad \wp_{22}(\mathbf{u})=x_{1}+x_{2}, \quad \wp_{221}(\mathbf{u})=\frac{x_{1} y_{2}-x_{2} y_{1}}{x_{1}-x_{2}}, \quad \wp_{222}(\mathbf{u})=\frac{y_{1}-y_{2}}{x_{1}-x_{2}}
$$

(see e.g. [12]). Thus the connection with the Bolza polynomial immediately leads to the solution of the Hénon-Heiles system in terms of Kleinian $\wp$ functions, which is

$$
q_{1}^{2}=16 \wp_{12}(\mathbf{u}), \quad q_{2}=-2 \wp_{22}(\mathbf{u})-2 a, \quad q_{1} p_{1}=8 \wp_{221}(\mathbf{u}), \quad p_{2}=-2 \wp_{222}(\mathbf{u}) .
$$

It is shown in 21] that the case (ii) Hénon-Heiles system has a Bäcklund transformation (BT) with parameter $\lambda$, which is a symplectic map with generating function $F\left(q_{1}, \tilde{q}_{1} ; \lambda\right)$ such that $d F=\sum_{j=1,2} p_{j} \mathrm{~d} q_{j}-\tilde{p}_{j} \mathrm{~d} \tilde{q}_{j}$. The explicit form of the generating function is

$F=Z+\frac{m}{2} \log \left(\frac{Z-m}{Z+m}\right)+\frac{16}{5} Y^{5}+4\left(q_{2}+\tilde{q}_{2}\right) Y^{3}+\left(2\left(q_{2}+\tilde{q}_{2}\right)^{2}-2 q_{2} \tilde{q}_{2}+\frac{1}{2}\left(q_{1}^{2}+\tilde{q}_{1}^{2}\right)+2 c\right) Y$,

where $Z\left(q_{j}, \tilde{q}_{j}\right)$ and $Y\left(q_{j}, \tilde{q}_{j}\right)$ are defined by

$$
Z^{2}=m^{2}+\lambda q_{1}^{2} \tilde{q}_{1}^{2}, \quad Y^{2}=\lambda+a-\frac{1}{2}\left(q_{2}+\tilde{q}_{2}\right) .
$$

The BT can be realized as a similarity transformation on the Lax pair (discrete Lax equation)

$$
\tilde{\mathbf{L}} \mathbf{M}=\mathbf{L M}
$$

where $\tilde{\mathbf{L}}=\mathbf{L}\left(x ; \tilde{q}_{j}, \tilde{p}_{j}\right)$ and

$$
\mathbf{M}=\left(\begin{array}{cc}
-Y & Y^{2}+x-\lambda \\
1 & -Y
\end{array}\right)
$$

is the elementary Darboux matrix (see [40]). Clearly from (4.5) the BT preserves the spectrum of $\mathbf{L}$, and so maps solutions to solutions.

In fact, the BT was constructed in [21] by making use of the formulae for the Darboux transformation of the Schrödinger equation, since the components of $\Psi$ in the linear system (4.4) are given by $\Psi=\left(\psi, \partial_{2} \psi\right)^{T}$ with $\left(\partial_{2}^{2}+q_{2}\right) \psi=(x+a) \psi$. Then the quantity $Y$ appearing in the Darboux matrix can be given explicitly in terms of the Baker function $\Phi_{+}$defined in (3.3) as

$$
Y=\partial_{2} \log \Phi_{+} \equiv \partial_{2} \log \Phi(\mathbf{u} ; \lambda)=\zeta_{2}(\mathbf{u}-\mathbf{v})-\zeta_{2}(\mathbf{u})+f_{\infty}^{(\lambda, \mu)} \mathrm{d} r_{2},
$$

and by a simple calculation using (3.5) we also have

$$
Y=\frac{\mu+\partial_{2} \mathcal{P}(\lambda ; \mathbf{u})}{2 \mathcal{P}(\lambda ; \mathbf{u})}=\frac{-\mu+\partial_{2} \mathcal{P}(\lambda ; \mathbf{u}-\mathbf{v})}{2 \mathcal{P}(\lambda ; \mathbf{u}-\mathbf{v})},
$$

which is equivalent to equation (2.9) in [21].

It follows from general results in [28] that the BT defined by the Darboux matrix (4.6) just gives a shift on the Jacobian, and here we see that

$\tilde{q}_{1}^{2}=16 \wp_{12}(\mathbf{u}-\mathbf{v}), \quad \tilde{q}_{2}=-2 \wp_{22}(\mathbf{u}-\mathbf{v})-2 a, \quad \tilde{q}_{1} \tilde{p}_{1}=8 \wp_{221}(\mathbf{u}-\mathbf{v}), \quad \tilde{p}_{2}=-2 \wp_{222}(\mathbf{u}-\mathbf{v})$. 
In terms of divisors, we have the equivalence

$$
\tilde{\mathcal{D}} \sim \mathcal{D}_{0}+(\lambda,-\mu)-\infty \sim\left(\tilde{x}_{1}, \tilde{y}_{1}\right)+\left(\tilde{x}_{2}, \tilde{y}_{2}\right)-2 \infty \sim \mathcal{D}_{-1}
$$

Similarly, applying the same BT but using the Baker function $\Phi_{-}$corresponds to adding the point $P=(\lambda, \mu)$ instead of $\hat{P}=(\lambda,-\mu)$, which gives the divisor $\mathcal{D}_{1} \sim \mathcal{D}_{0}+(\lambda, \mu)-\infty$. In other words, the $n$th term in the sequence of Bolza polynomials (3.14), with argument $\mathbf{u}+n \mathbf{v} \in \operatorname{Jac}(X)$, is the result of $n$ applications of the Hénon-Heiles BT, adding the same point $P$ each time. We should point out that the Hénon-Heiles BT given here and in [21] is the same as the $g=2$ discrete odd Mumford system in [28], with a particular choice of symplectic structure.

\section{Conclusions}

We have constructed an eighth order bilinear recurrence relation (3.11) whose $n$th term is expressed in terms of the Kleinian sigma function of an odd hyperelliptic curve (3.1) of genus two. Moreover, we have explained how this is connected to the BT for the case (ii) integrable Hénon-Heiles system of [21, 22: each shift $n \rightarrow n+1$ in the recurrence corresponds to the shift on the Jacobian induced by the BT.

The question remains of whether we can solve the initial value problem for an eighth order recurrence of the form (3.11). In fact, the expression (3.10) depends on at most 10 parameters: the five coefficients $c_{j}, j=0, \ldots, 4$ that specify the curve plus the three points $P_{1}, P_{2}, P$ on the curve to specify the sequence of divisors $\mathcal{D}_{n} \sim\left(P_{1}-\infty\right)+\left(P_{2}-\infty\right)+n(P-\infty)$, and the two prefactors $A, B$. (Actually, the constant $c_{4}$ can be removed from the start by making a shift in $x$.) The general eighth order recurrence (3.11) would have four parameters $\alpha_{j}, j=0,1,2,3$ in general position and 8 initial data $\tau_{j}, j=0, \ldots, 7$. In fact, by counting arguments, we expect that the general solution of a bilinear recurrence consisting of $N+2$ bilinear terms,

$$
\tau_{n+N+1} \tau_{n-N-1}=\sum_{j=0}^{N} \alpha_{j} \tau_{n+j} \tau_{n-j}
$$

which is of order $2 N+2$, should correspond to a sequence of divisors $\mathcal{D}_{n} \sim \sum_{j=1}^{N}\left(P_{j}-\infty\right)+$ $n(P-\infty)$ on an hyperelliptic curve of genus $N$, since this solution should depend on $3 N+3$ parameters. This expectation agrees with the form of vector addition formulae for Riemann theta functions obtained by Buchstaber and Krichever [7], which have precisely $N+2$ terms in genus $N$. However, preliminary calculations suggest the need to consider a different model for these curves, without a branch point at infinity. For example, sigma functions for curves of the form

$$
y^{2}=4 x^{6}+\sum_{j=0}^{5} c_{j} x^{j}
$$

should give solutions to the Somos 6 recurrence

$$
\tau_{n+3} \tau_{n-3}=\sum_{j=0}^{2} \alpha_{j} \tau_{n+j} \tau_{n-j} ;
$$

this should correspond to the BT for the $g=2$ even Mumford system in [28, which has a spectral curve of this type. This agrees with recent results of van der Poorten [35], which show that a certain class of Somos 6 sequences arise from the continued fraction expansion of the square root of a sextic. 
Note added in proof. In a private communication, David Cantor has shown one of us that by extending the results of [1] to divisor sequences $\mathcal{D}_{0}+n(P-\infty)$ it is possible to prove that the sequence of tau functions $\tau_{n}$ given by (3.10) in Theorem 2 satisfies a family of trilinear recurrence relations given in terms of a Hankel type determinant, that is

$$
a_{2}^{2} a_{m} \tau_{n} \tau_{n+m} \tau_{n-m}=\left|\begin{array}{ccc}
a_{m} \tau_{n-2} & a_{m+1} \tau_{n-1} & a_{m+2} \tau_{n} \\
a_{m-1} \tau_{n-1} & a_{m} \tau_{n} & a_{m+1} \tau_{n+1} \\
a_{m-2} \tau_{n} & a_{m-1} \tau_{n+1} & a_{m} \tau_{n+2}
\end{array}\right|
$$

for all $m, n \in \mathbb{Z}$, where

$$
a_{m}=\frac{\sigma(m \mathbf{v})}{\sigma_{2}(\mathbf{v})^{m^{2}}} .
$$

The above formula is the genus two analogue of a family of higher recurrences satisfied by Somos 4 sequences [36] (cf. also Morgan Ward's identity (2.6) for elliptic divisibility sequences). Up to a suitable choice of normalization, these $a_{m}$ are the genus two division polynomials derived by Cantor [11, whose functional form has been specified precisely in the works 29, 30] of Matsutani, where they are referred to as hyperelliptic psi-functions. In the limit $\mathbf{u} \rightarrow 0$, when $\tau_{n} \rightarrow a_{n}$, the equation (5.1) reduces to a Hankel formula for these psi-functions obtained by algebraic means in [11, which is also presented as formula (3.10) in [29], and rederived using analytic techniques in [30. Setting $m=3$ in (5.1) yields a sixth order trilinear recurrence for $\tau_{n}$, while setting $m=4$ yields an eighth order trilinear recurrence. As was first pointed out to us by Cantor, the bilinear recurrence (3.11) above then follows as a consequence of these two trilinear identities, upon eliminating between the two of them. Interestingly, Buchstaber and Leykin have very recently derived a trilinear differential addition formula for genus two sigma functions - see Theorem 5.6 in [10].

Acknowledgments. AH is grateful to the University of Kent for supporting the project Algebraic curves and functional equations in mathematical physics with a Colyer-Fergusson Award, which funded his visits to Edinburgh. AH also thanks the following people: Graham Everest for introducing him to the arithmetic of quadratic recurrences; Christine Swart for providing a copy of her thesis [43; and Vadim Kuznetsov, Pol Vanhaecke and Orlando Ragnisco for many enlightening conversations about BTs. Finally, VZE and AH are very grateful to Shigeki Matsutani for providing copies of his papers, and for making some very insightful comments about our work.

\section{References}

[1] M. Adler and J. Moser, On a Class of Polynomials connected with the Korteweg-deVries Equation, Commun. Math. Phys. 61 (1978) 1-30.

[2] H.F. Baker, An introduction to the theory of Multiply Periodic Functions, Cambridge University Press (1907).

[3] G. Bastien and M. Rogalski, On some algebraic difference equations $u_{n+2} u_{n}=\psi\left(u_{n+1}\right)$ in $\mathbb{R}_{*}^{+}$ related to families of conics or cubics: generalization of the Lyness' sequences, J. Math. Anal. Appl. 300 (2004) 303-333.

[4] E.D. Belokolos, V.Z. Enolskii and M. Salerno, Wannier functions for quasi-periodic finite-gap potentials, preprint math-ph/0412081 (2004).

[5] O. Bolza, On the first and second derivatives of hyperelliptic $\sigma$-functions, Amer. J. Math 17 (1895), no. 11. 
[6] M. Bruschi, O. Ragnisco, P.M. Santini and G.-Z. Tu, Integrable Symplectic Maps, Physica D 49 (1991) 273-294.

[7] V.M. Buchstaber and I.M. Krichever, Vector Addition Theorems and Baker-Akhiezer Functions, Teoret. Mat. Fiz. 94 (1993) 200-212.

[8] V.M. Buchstaber, V.Z. Enolskii and D.V. Leykin, Hyperelliptic Kleinian functions and applications, in 'Solitons, Geometry and Topology: On the Crossroad,' (eds. V.M. Buchstaber and S.P. Novikov), AMS Translations Series 2, Vol. 179, AMS (1997) 1-34.

[9] V.M. Buchstaber, V.Z. Enolskii and D.V. Leykin, Rational analogues of abelian functions, Funksional. Anal. Pril. 33, no.2 (1999) 1-15; translation in Func. Anal. Appl. 33, no.2 (1999) 83-94.

[10] V.M. Buchstaber and D.V. Leykin, Hyperelliptic Addition Law, J. Nonlin. Math. Phys. 12, Suppl. 1 (2005) 106-123.

[11] D. Cantor, On the analogue of the division polynomials for hyperelliptic curves, J. reine angew. Math. 447 (1994) 91-145.

[12] J.C. Eilbeck, V.Z. Enolskii and E. Previato, On a generalized Frobenius-Stickelberger Addition Formula, Lett. Math. Phys. 63 (2003) 5-17.

[13] M. Einsiedler, G. Everest and T. Ward, Primes in elliptic divisibility sequences, LMS Journal of Computation and Mathematics 4 (2001) 1-13.

[14] V.Z. Enolskii and J. Gibbons, Addition on strata of the theta-divisor and integrable systems, preprint (2004).

[15] G. Everest, V. Miller and N. Stephens, Primes generated by elliptic curves, Proc. Amer. Math. Soc. 132 (2003) 955-963.

[16] G. Everest, A. van der Poorten, I. Shparlinski and T. Ward, Recurrence Sequences, AMS Mathematical Surveys and Monographs, vol. 104, American Mathematical Society (2003).

[17] A.P. Fordy, The Hénon-Heiles system revisited, Physica D 52 (1991) 204-210.

[18] S. Fomin and A. Zelevinsky, The Laurent Phenomenon, Adv. Appl. Math. 28 (2002) 119-144.

[19] R. Hirota, Exact solution of the Korteweg-deVries equation for multiple collision of solitons, Phys. Rev. Lett. 27 (1972) 1192-1194.

[20] A.N.W. Hone, Exact discretization of the Ermakov-Pinney equation, Phys. Lett. A263 (1999) $347-354$.

[21] A.N.W. Hone, V.B. Kuznetsov and O. Ragnisco, Bäcklund transformations for many-body systems related to KdV, J. Phys. A: Math. Gen. 32 (1999) L299-L306.

[22] A.N.W. Hone, V.B. Kuznetsov and O. Ragnisco, Bäcklund transformations for the HénonHeiles and Garnier systems, in 'Proceedings of the SIDE III meeting, Sabaudia 1998' (eds. D. Levi and O. Ragnisco), CRM Proceedings and Lecture Notes Series 25, American Mathematical Society (2000) 231-235.

[23] A.N.W. Hone, Algebraic curves, integer sequences and a discrete Painlevé transcendent, poster at SIDE 6, Helsinki, Finland, 19-24 June 2004; preprint (2004) UKC/IMS/04/20, University of Kent. 
[24] A.N.W. Hone, Elliptic curves and quadratic recurrence sequences, Bull. Lond. Math. Soc. 37 (2005) 161-171.

[25] A.N.W. Hone, Sigma function solution of the initial value problem for Somos 5 sequences; preprint math.NT/0501554

[26] V.G. Kac, Infinite Dimensional Lie Algebras, Birkhäuser, Boston (1983).

[27] I.M. Krichever, P. Wiegmann and A. Zabrodin, Elliptic solutions to difference non-linear equations and related many-body problems, Comm. Math. Phys. 193 (1998) 373-396.

[28] V. Kuznetsov and P. Vanhaecke, Bäcklund transformations for finite-dimensional integrable systems: a geometric approach, J. Geom. Phys. 44 (2002) 1-40.

[29] S. Matsutani, Elliptic and hyperelliptic solutions to discrete Painlevé I and its extension to higher order difference equations, Phys. Lett. A 300 (2002) 233-242.

[30] S. Matsutani, Recursion relation of hyperelliptic PSI-functions of genus two, Int. Transforms Spec. Func. 14 (2003) 517-527.

[31] Y. Ohta, J. Satsuma, D. Takahashi and T. Tokihiro, An Elementary Introduction to Sato Theory, Prog. Theor. Phys. Suppl. 94 (1988) 210-241.

[32] Y. Ohta, K.M. Tamizhmani, B. Grammaticos and A. Ramani, Singularity confinement and algebraic entropy: the case of the discrete Painlevé equations, preprint solv-int/9910007

[33] Y. Onishi, Determinant expressions for Abelian functions in genus two, Glasgow Math. J. 44 (2002) 353-364.

[34] Y. Onishi, Determinant expressions for hyperelliptic functions (with an Appendix by Shigeki Matsutani: Connection of the formula of Cantor and of Brioschi-Kiepert type), preprint (2004); Proc. Edinburgh Math. Soc., to appear.

[35] A. van der Poorten, Genus 2 curves, continued fractions, and Somos sequences, preprint math.NT/0412372

[36] A. van der Poorten and C.S. Swart, Recurrence Relations for Elliptic Sequences: every Somos 4 is a Somos k, preprint math.NT/0412293

[37] G.R.W. Quispel, J.A.G. Roberts and C.J. Thompson, Integrable mappings and soliton equations II, Physica D 34 (1989) 183-192.

[38] A. Ramani, B. Grammaticos and J. Satsuma, Bilinear discrete Painlevé equations, J. Phys. A: Math. Gen. 28 (1995) 4655-4665.

[39] R. Robinson, Periodicity of Somos sequences, Proc. Amer. Math. Soc. 116 (1992) 613-619.

[40] A.B. Shabat and A.P. Veselov, Dressing chain and spectral theory of the Schrödinger operator, Funct. Anal. Appl. 27:2 (1993) 1-21.

[41] R. Shipsey, Elliptic divisibility sequences, PhD thesis, University of London (2000).

[42] N.J.A. Sloane, On-Line Encyclopedia of Integer Sequences, http://www.research.att.com/〜njas/sequences, sequence A006720.

[43] C.S. Swart, Elliptic curves and related sequences, PhD thesis, University of London (2003).

[44] A.P. Veselov, Integrable maps, Russian Math. Surveys 46 (1991) 1-51. 
[45] M. Ward, Memoir on Elliptic Divisibility Sequences, Amer. J. Math. 70 (1948) 31-74.

[46] M. Ward, The Law of Repetition of Primes in an Elliptic Divisibility Sequence, Duke Math. J. 15 (1948) 941-946.

[47] E.T. Whittaker and G.N. Watson, A Course of Modern Analysis, 4th edition, Cambridge (1965). 